\title{
Comparative analysis of root transcriptomes from two contrasting drought-responsive Williams 82 and DT2008 soybean cultivars under normal and dehydration conditions
}

\section{OPEN ACCESS}

Edited by:

Girdhar Kumar Pandey,

Delhi University, India

Reviewed by:

Asif Ashfaq Khan,

Temasek Life Sciences Laboratories,

Singapore

Ratna Karan

University of Florida, USA

*Correspondence:

Lam-Son Phan Tran,

Signaling Pathway Research Unit,

RIKEN Center for Sustainable

Resource Science, 1-7-22

Suehiro-cho, Tsurumi-ku, Yokohama

230-0045, Japan

son.tran@riken.jp

Specialty section:

This article was submitted to

Plant Physiology,

a section of the journal

Frontiers in Plant Science

Received: 22 May 2015

Accepted: 06 July 2015

Published: 07 August 2015

Citation:

Ha CV, Watanabe Y, Tran UT, Le DT,

Tanaka M, Nguyen KH, Seki M,

Nguyen DV and Tran L-SP (2015)

Comparative analysis of root transcriptomes from two contrasting drought-responsive Williams 82 and

DT2008 soybean cultivars under normal and dehydration conditions.

Front. Plant Sci. 6:551.

doi: $10.3389 /$ fpls.2015.00551

\author{
Chien Van $\mathrm{Ha}^{1,2}$, Yasuko Watanabe ${ }^{1}$, Uyen Thi Tran ${ }^{1}$, Dung Tien Le ${ }^{2}$, Maho Tanaka ${ }^{3}$, \\ Kien Huu Nguyen ${ }^{1,2}$, Motoaki Seki ${ }^{3,4}$, Dong Van Nguyen ${ }^{2}$ and Lam-Son Phan Tran ${ }^{1 *}$ \\ ${ }^{1}$ Signaling Pathway Research Unit, RIKEN Center for Sustainable Resource Science, Yokohama, Japan, ${ }^{2}$ National Key \\ Laboratory for Plant Cell Technology, Agricultural Genetics Institute, Vietnamese Academy of Agricultural Science, Hanoi, \\ Vietnam, ${ }^{3}$ Plant Genomic Network Research Team, RIKEN Center for Sustainable Resource Science, Yokohama, Japan, \\ ${ }^{4}$ CREST, Japan Science and Technology Agency, Kawaguchi, Japan
}

The economically important DT2008 and the model Williams 82 (W82) soybean cultivars were reported to have differential drought-tolerant degree to dehydration and drought, which was associated with root trait. Here, we used 66K Affymetrix Soybean Array GeneChip to compare the root transcriptomes of DT2008 and W82 seedlings under normal, as well as mild ( $2 \mathrm{~h}$ treatment) and severe ( $10 \mathrm{~h}$ treatment) dehydration conditions. Out of the 38172 soybean genes annotated with high confidence, 822 (2.15\%) and $632(1.66 \%)$ genes showed altered expression by dehydration in W82 and DT2008 roots, respectively, suggesting that a larger machinery is required to be activated in the drought-sensitive W82 cultivar to cope with the stress. We also observed that long-term dehydration period induced expression change of more genes in soybean roots than the short-term one, independently of the genotypes. Furthermore, our data suggest that the higher drought tolerability of DT2008 might be attributed to the higher number of genes induced in DT2008 roots than in W82 roots by early dehydration, and to the expression changes of more genes triggered by short-term dehydration than those by prolonged dehydration in DT2008 roots vs. W82 roots. Differentially expressed genes (DEGs) that could be predicted to have a known function were further analyzed to gain a basic understanding on how soybean plants respond to dehydration for their survival. The higher drought tolerability of DT2008 vs. W82 might be attributed to differential expression in genes encoding osmoprotectant biosynthesis-, detoxification- or cell wall-related proteins, kinases, transcription factors and phosphatase $2 \mathrm{C}$ proteins. This research allowed us to identify genetic components that contribute to the improved drought tolerance of DT2008, as well as provide a useful genetic resource for in-depth functional analyses that ultimately leads to development of soybean cultivars with improved tolerance to drought.

Keywords: soybean, dehydration, root microarray, differential expression, differential drought tolerability 


\section{Introduction}

Soybean (Glycine max L.) has been regarded as one of the major legume crops worldwide with multibillion dollars in value. Its seed product provides a substantial source of vegetable protein and oil, micronutrients and minerals for animal feed and human consumption (Tran and Nguyen, 2009; Choudhary and Tran, 2011). In the last several years, soybean has also shown its increasing importance in industry by supplying materials for production of biodiesel, plastics, lubricants, and hydraulic fluids (Hsien, 2015). Unfortunately, like many other crops, soybean's growth and development, and thus its productivity, are severely affected by various environmental stresses, especially drought that can cause yield loss by approximately $11-50 \%$ in various countries, including Vietnam (Vinh et al., 2010; Sadeghipour and Abbasi, 2012; Ferreira Neto et al., 2013; Ku et al., 2013). Thus, in recent years, scientific community has paid a great attention to research toward understanding of mechanisms underlying soybean responses to drought, ultimately leading to development of improved drought-tolerant soybean cultivars (Tran and Mochida, 2010; Thao and Tran, 2012; Hossain et al., 2013; Deshmukh et al., 2014).

In general, to cope with drought, a number of adaptive mechanisms are activated in plants, including soybean, through various signal transduction pathways which lead to the activation of various molecular, biochemical, and physiological responses (Hadiarto and Tran, 2011; Ha et al., 2012; Hossain et al., 2013; Deshmukh et al., 2014; Karan and Subudhi, 2014; Khan et al., 2014). Studies of the mechanisms regulating these adaptive responses, as well as identification of genes involved in these mechanisms have become a great interest of the research community. Recent advances in omics technologies, especially transcriptomics, have enabled us to identify genes, gene families and pathways associated with plant responses to stresses in a systematic manner (Ma et al., 2012; Jogaiah et al., 2013; Deshmukh et al., 2014). Taking advantage of the available soybean genomic sequences and recent progress in microarray technologies (Schmutz et al., 2010; Mochida and Shinozaki, 2011), the 66K Affymetrix soybean array platform has been designed by a US consortium, which allows us to study the expression of all the putatively annotated genes in soybean at different developmental stages, under normal, abiotic, and biotic stress conditions in a relatively reliable manner (Valdes-Lopez et al., 2011; Le et al., 2012b; Wan et al., 2015).

Root development and plasticity have been identified as a key trait in plant adaptation to drought as they determine plant access to soil water. For instance, longer primary root and/or larger xylem diameters in deep roots and/or larger lateral root system are desirable root traits which help plants adapt better to drought by acquiring water from lower soil layers or foraging subsoil surface moisture (Manavalan et al., 2009; Comas et al., 2013). Thus, identification of quantitative trait loci and genes involved in determination of root traits has been regarded as an important task of research community that has interest in elucidation of molecular mechanisms regulating plant responses to drought (Manavalan et al., 2009; Comas et al., 2013; Thao et al., 2013; Satbhai et al., 2015).
In this report, we used the $66 \mathrm{~K}$ Affymetrix soybean GeneChip to study (i) the transcriptome-wide changes in soybean dehydrated roots vs. non-dehydrated roots and (ii) analyze the genome-wide differential gene expression in the root tissues of Williams 82 (W82) and DT2008, which have differential dehydration/drought-responsive phenotype (Ha et al., 2013), under normal and dehydration conditions. W82 is a model cultivar whose genome was sequenced several years ago (Schmutz et al., 2010), while DT2008 is an economically important cultivar grown in many regions of Vietnam (Vinh et al., 2010). DT2008 was reported to display stronger tolerance to drought than W82 in a comparative analysis, which might be associated with a better root trait (Ha et al., 2013). The results of this study will enable us to identify dehydration-responsive genes in soybean roots and understand the genetic network underlying the differential drought tolerability of W82 and DT2008, as well as provide us with a list of promising candidate genes that hold potential application in development of improved drought-tolerant transgenic soybean varieties through genetic engineering.

\section{Results}

\section{Microarray Analysis of W82 and DT2008 Root Transcriptomes under Normal and Dehydration Conditions}

In our experimental design, root transcriptomes of droughtsensitive W82 and drought-tolerant DT2008 were compared at 0 (unstressed), 2 (early stress), and $10 \mathrm{~h}$ (late stress) of dehydration (Figure 1A) by microarray analysis using the $66 \mathrm{~K}$ soybean GeneChip (Supplementary Table S1). The relative water content (RWC) of dehydrated plants was measured during dehydration treatment, and the values were 70.2 and $75.8 \%$ for W82 and DT2008, respectively, at $2 \mathrm{~h}$, whereas the respective values at $10 \mathrm{~h}$ of dehydration were 18.1 and $40 \%$ (Figure 1B), indicating the mild and severe stress intensities. This experimental design thus allowed us to identify (i) dehydration-responsive genes in each cultivar in a time-course manner (W-D2/W-C and $\mathrm{W}$ D10/W-C; DT-D2/DT-C and DT-D10/DT-C), as well as (ii) genes involved in regulatory network that regulates differential root trait (DT-C/W-C, DT-D2/ W-D2, and DT-D10/W-D10), thereby potentially contributing to higher drought tolerance of DT2008 relative to $\mathrm{W} 82$.

Recently, the soybean genome sequence and its annotation have been substantially improved in the newest version Glyma v2.0 [Glyma.Wm82.a2.v1 (genome assembly 2 annotation version 1)] released by Phytozome 10.1 (http://phytozome.jgi. doe.gov/pz/portal.html). Using this latest Glyma v2.0 annotation, the $66 \mathrm{~K}$ soybean GeneChip allowed us to study the expression of 38172 genes with high confidence. These genes were subjected to a search for differentially expressed genes (DEGs) using the criterion of two-fold expression change $(q<0.05)$ (Supplementary Table S2). We found that 105 and 526 genes were upregulated and 47 and 215 were downregulated in W82 roots treated with dehydration for 2 and $10 \mathrm{~h}$, respectively 

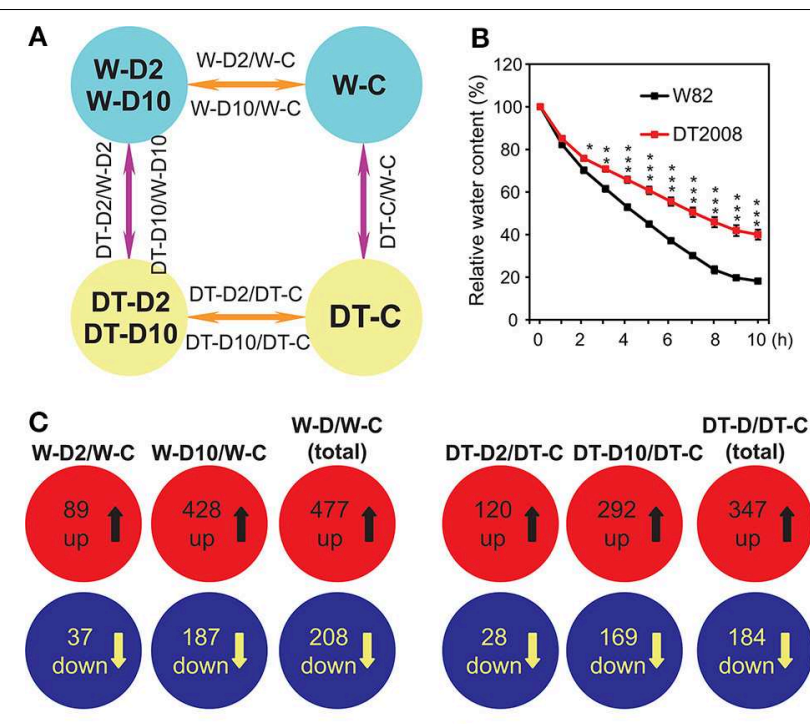

W-D/W-C

(total)

D

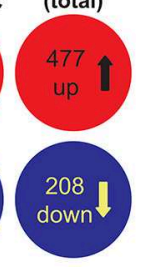

W-D2/W-C

W-D10/W-C
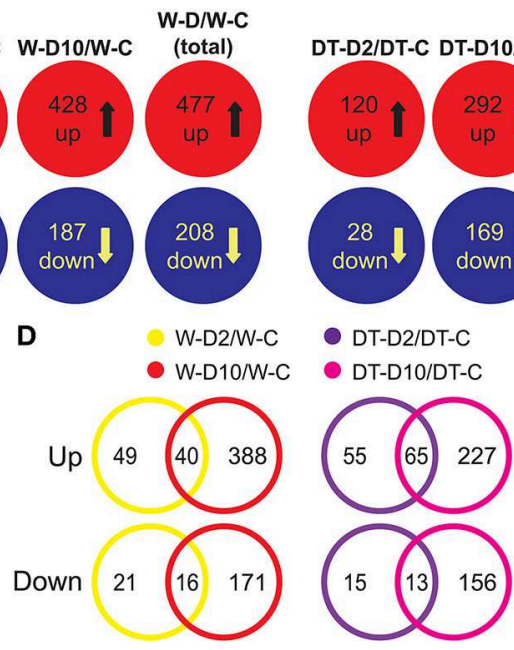

DT-D/DT-C

C (total) microarray analysis. (A) Diagrams showing experimental design and comparisons. (B) Relative water content of W82 and DT2008 plants exposed to a dehydration treatment. Data represent the mean and SE $(n=5)$. Asterisks indicate significant differences as determined by a Student's $t$-test $\left({ }^{*} P<0.05\right.$; ${ }^{\star \star} P<0.01$ and $\left.{ }^{{ }^{\star \star}} P<0.001\right)$. (C) Upregulated and downregulated genes identified in each comparison from 34097 genes that were assigned with a putative function. Data were obtained from the results of three independent microarray experiments of three biological repeats. (D) Effect of stress intensity on gene expression in roots of W82 and DT2008 as indicated by Venn analysis of differentially expressed gene sets identified in (C). W-D2NW-C,

W82-dehydrated-2 $\mathrm{h}$ vs. W82-well-watered control-0 $\mathrm{h}$; W-D10/W-C, W82-dehydrated-10 $\mathrm{h}$ vs. W82-well-watered control- $0 \mathrm{~h}$; W-D/W-C represents W-D2/W-C and/or W-D10N-C (W82-dehydrated-2 $\mathrm{h}$ and/or $10 \mathrm{~h}$ vs. W82-well-watered control-0 h); DT-D2/DT-C, DT2008-dehydrated-2 $\mathrm{h}$ vs. DT2008-well-watered control-0 h; DT-D10/DT-C, DT2008-dehydrated-10 h vs. DT2008-well-watered control-0 h; DT-D/DT-C represents DT-D2/DT-C and/or DT-D10/DT-C (DT2008-dehydrated-2 h and/or $10 \mathrm{~h}$ vs. DT2008-well-watered control-0 h).

(Supplementary Figure S1A, comparisons W-D2/W-C and WD10/W-C; Supplementary Tables S3A-D), whereas 131 and 355 genes were upregulated and 34 and 199 were downregulated in 2 and 10 h-dehydrated DT2008 roots vs. control, respectively (Supplementary Figure S1A, comparisons DT-D2/DT-C and DTD10/DT-C; Supplementary Tables S4A-D). A Venn analysis indicated that 50 genes were upregulated in both $2 \mathrm{~h}$ - and 10 h-dehydrated W82 roots, whereas 55 were upregulated by $2 \mathrm{~h}$ dehydration and 476 genes by $10 \mathrm{~h}$ dehydration only (Supplementary Figures S1A,B; Supplementary Table S3E), making a total of 581 unique genes upregulated by at least one dehydration treatment (Supplementary Figure S1A, W-D/W-C).
Similarly, we found an overlap of 21 downregulated genes in roots of W82 treated with dehydration for 2 and $10 \mathrm{~h}$, and a list of 241 unique genes downregulated in dehydrated W82 roots under these two treatment conditions (Supplementary Figures S1A,B; Supplementary Table S3F). As for the drought-tolerant DT2008, we noted from the Venn diagrams that 71 upregulated and 16 downregulated genes were overlapped between DT-D2/DTC and DT-D10/DT-C comparisons, while totally 415 and 217 unique genes were upregulated and downregulated, respectively, in 2 and/or 10 h-dehydrated DT2008 roots (Supplementary Figures S1A,B, comparison DT-D/DT-C; Supplementary Tables S4E,F).

\section{Identification of Dehydration-responsive Genes with Putative Function in W82 and DT2008 Roots}

Next, to identify genes modulated by dehydration in roots of W82 and/or DT2008, which have a predicted function for subsequent comparative analyses, we removed the genes with "no original description," which are a total of 4075 genes, and examined only 34097 genes that could be assigned with a putative function (Supplementary Table S5). This approach allowed us to link the expression change by stress treatment with gene function, thereby enabling us to explain the differential root responses of W82 and DT2008 to drought. We noted 89 and 428 upregulated genes and 37 and 187 downregulated genes in W-D2/W-C and W-D10/W-C comparisons, respectively (Figure 1C; Supplementary Tables S6A-D). At the same time, we were able to detect 120 and 292 upregulated genes and 28 and 169 downregulated genes in DT-D2/DT-C and DTD10/DT-C comparisons, respectively (Figure 1C; Supplementary Tables S7A-D). As shown by Venn analysis, 40 upregulated and 16 downregulated genes were overlapped between $\mathrm{W}$ D2/W-C and W-D10/W-C comparisons, while a total of 477 and 208 unique genes were found to be upregulated and downregulated, respectively, in dehydrated W82 roots (Figure 1D; Supplementary Tables S6E,F). In case of DT2008, we detected 65 and 13 overlapped genes in the upregulated and downregulated gene sets obtained from DT-D2/DT-C and DT-D10/DT-C comparisons. Removing the overlapped genes made the lists of unique genes upregulated (347) or downregulated (184) by at least one dehydration treatment in DT2008 roots vs. control for further analyses (Figures 1C,D; Supplementary Tables S7E,F). Several genes showing various degrees of induction and repression by dehydration were selected for verification of the microarray data using real-time quantitative PCR (RT-qPCR) (Supplementary Table S8). Results shown in Figure 2 clearly demonstrated the reliability of the microarray data.

\section{Distribution of the Dehydration-responsive Gene Sets Identified in W82 and DT2008 Roots into Functional Categories}

As a means to understand the molecular mechanisms underlying root responses that soybean plants develop to increase their adaptation to drought, we used MapMan to classify the dehydration-responsive genes detected in W82 and DT2008 into various functional categories. Lists of unique genes with 
A

\begin{tabular}{|c|c|c|c|c|c|c|c|c|c|c|c|c|c|c|}
\hline \multirow{3}{*}{ Glyma 2 ID } & \multicolumn{14}{|c|}{ Microarray data } \\
\hline & \multicolumn{2}{|c|}{ DT-C vs W-C } & \multicolumn{2}{|c|}{ DT-D2 vs W-D2 } & \multicolumn{2}{|c|}{ DT-D10 vs W-D10 } & \multicolumn{2}{|c|}{ W-D2 vs W-C } & \multicolumn{2}{|c|}{ W-D10 vs W-C } & \multicolumn{2}{|c|}{ DT-D2 vs DT-C } & \multicolumn{2}{|c|}{ DT-D10 vs DT-C } \\
\hline & $\begin{array}{c}\text { Fold } \\
\text { change }\end{array}$ & q-value & $\begin{array}{c}\text { Fold } \\
\text { change }\end{array}$ & q-value & $\begin{array}{c}\begin{array}{c}\text { Fold } \\
\text { change }\end{array} \\
\end{array}$ & q-value & $\begin{array}{c}\text { Fold } \\
\text { change }\end{array}$ & & $\begin{array}{c}\begin{array}{c}\text { Fold } \\
\text { change }\end{array} \\
\end{array}$ & q-value & $\begin{array}{c}\text { Fold } \\
\text { change }\end{array}$ & q-value & $\begin{array}{c}\begin{array}{c}\text { Fold } \\
\text { change }\end{array} \\
\end{array}$ & q-value \\
\hline Glyma.04G083000 & 2.614555 & 0.042432 & 3.258701 & 0.050831 & \begin{tabular}{|l|}
1.17867 \\
\end{tabular} & 0.595939 & 6.755826 & 0.039775 & 14.97512 & & 8.420253 & 0.037245 & 6.750947 & \\
\hline Glyma.04 & 5.388354 & 0.041824 & 8.15626 & 0.037069 & 2.222395 & 19264 & 2.316769 & 0.088876 & 7.598483 & 0.024694 & 3.506853 & 0.097159 & 3.133949 & 0.064747 \\
\hline Glyma.1 & -4.28059 & 0.101151 & -2.9485 & 0.034435 & -1.93853 & 0.169071 & -1.6523 & 0.103328 & -3.32594 & 0.03359 & -1.13811 & 0.851662 & -1.5062 & 0.47248 \\
\hline Glyma.13G030900 & 3.141957 & 0.025417 & 2.730304 & 0.05332 & 2.092875 & 0.149397 & 1.729662 & 0.132444 & 2.142148 & 0.071613 & 1.503046 & 0.114282 & 1.426897 & 0.066656 \\
\hline Glyma.13G095200 & -1.91738 & 0.205791 & 2.155244 & 0.040768 & 1.032759 & 0.884863 & -1.81058 & 0.221835 & 2.091222 & 0.129926 & 2.282378 & 0.057367 & 4.141017 & 0.02328 \\
\hline Glyma.13G279900 & 1.248811 & 0.395151 & 1.521326 & 0.073822 & 1.124983 & 0.643191 & 1.964096 & 0.069524 & 3.092159 & 0.028103 & 2.3927 & 0.070246 & 2.785551 & 0.045294 \\
\hline Glyma.19G227800 & 3.071611 & 0.140748 & 3.704389 & 0.031099 & 1.096094 & 0.910573 & 4.933751 & 0.064345 & 46.86791 & 0.018625 & 5.950145 & 0.06752 & 16.72466 & 0.041343 \\
\hline
\end{tabular}

B

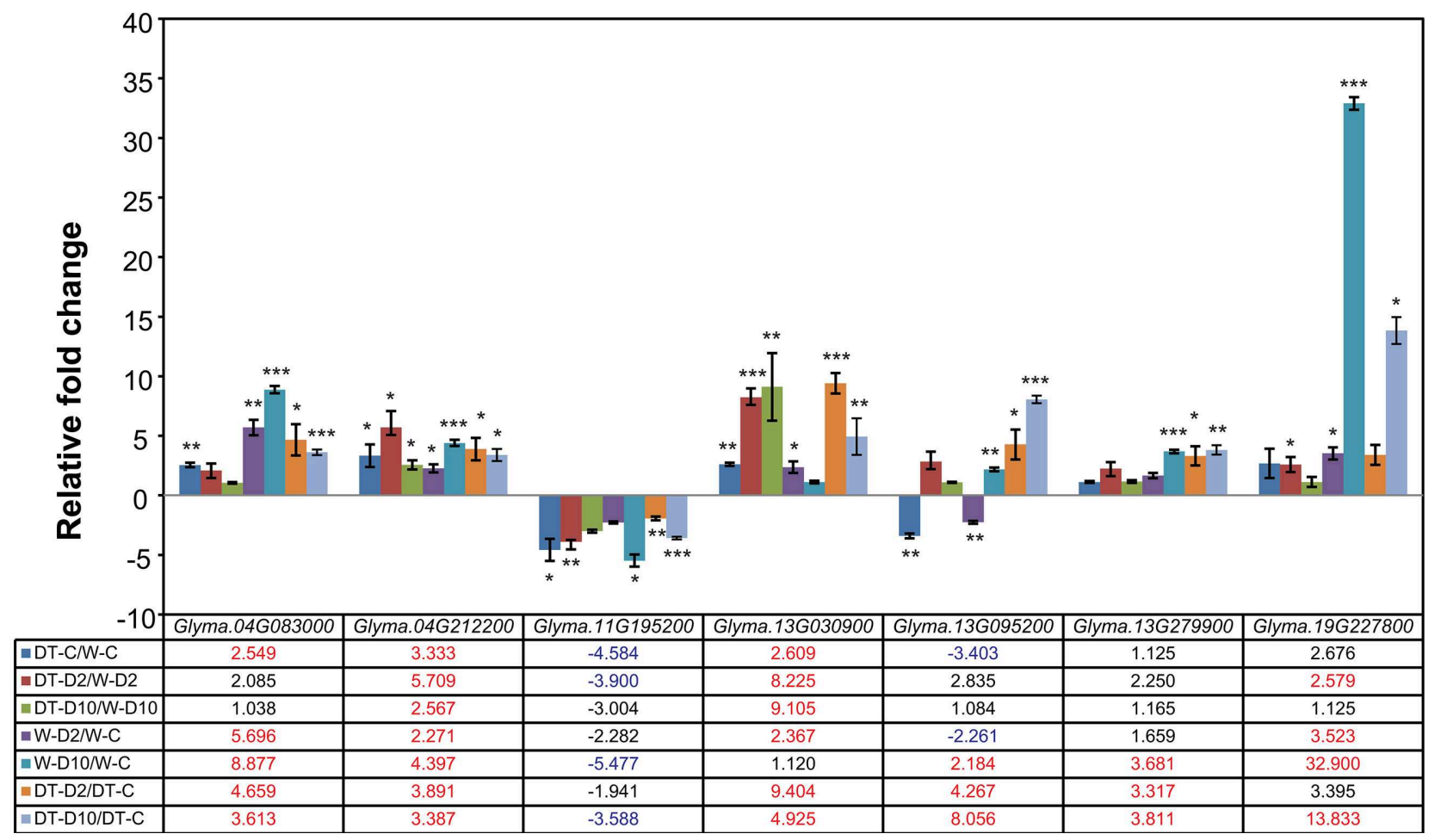

FIGURE 2 | Validation of microarray data by real-time quantitative PCR (RT-qPCR). Seven genes were selected for RT-qPCR verification of the microarray data. (A) Fold changes were obtained from microarray analysis. Red- and blue-color letters indicate absolute fold-changes $\geq 2$ with $q<0.05$. (B) Fold changes obtained by RT-qPCR of three independent biological replicates. The Fbox was used as reference gene. Data represent the mean plus SE $(n=3)$. Asterisks indicate significant differences as determined by a Student's $t$-test $\left({ }^{\star} P<0.05\right.$; ${ }^{\star \star} P<0.01$ and $\left.{ }^{\star * \star} P<0.001\right)$. Red- and blue-color letters indicate absolute fold-changes $\geq 2$ with $P<0.05$. W-D2/W-C, W82-dehydrated-2h vs. W82-well-watered control-0 h; W-D10/W-C, W82-dehydrated-10 h vs. W82-well-watered control-0 h; DT-D2/DT-C, DT2008-dehydrated-2 h vs.

DT2008-well-watered control-0 h; DT-D10/DT-C, DT2008-dehydrated-10 h vs. DT2008-well-watered control-0h. putatively predicted function (Supplementary Tables S6E,F, $\mathrm{S} 7 \mathrm{E}, \mathrm{F})$, which were found to be upregulated or downregulated in W82 or DT2008 roots by at least one dehydration treatment, either 2 or $10 \mathrm{~h}$ treatment, were assembled and subjected to MapMan analyses for assignment of each gene into functional category (Figure 3). Our data indicated that among the 20 most abundant categories, in both W82 and DT2008 roots, the upregulated genes of the TF category were the most highly enriched genes, whereas the downregulated genes were enriched in "protein synthesis, targeting, modification, etc" category.

\section{Brief Description of the Dehydration-responsive Gene Sets Identified in W82 and DT2008 Roots}

A closer look at the sets of the DEG sets identified in W82 and DT2008 roots under dehydration revealed a number of common phenomena between their up- and downregulated gene sets, respectively (comparisons $\mathrm{W}-\mathrm{D} / \mathrm{W}-\mathrm{C}$ and DT-D/DTC) (Supplementary Tables S6E,F, S7E,F). Many genes belonging to different TF families, such as the AP2_EREBP-, bZIP-, MYB- and NAC-type TF families, exhibited transcriptional changes by dehydration in both W82 and DT2008 roots, of which more dehydration-inducible genes were found than 


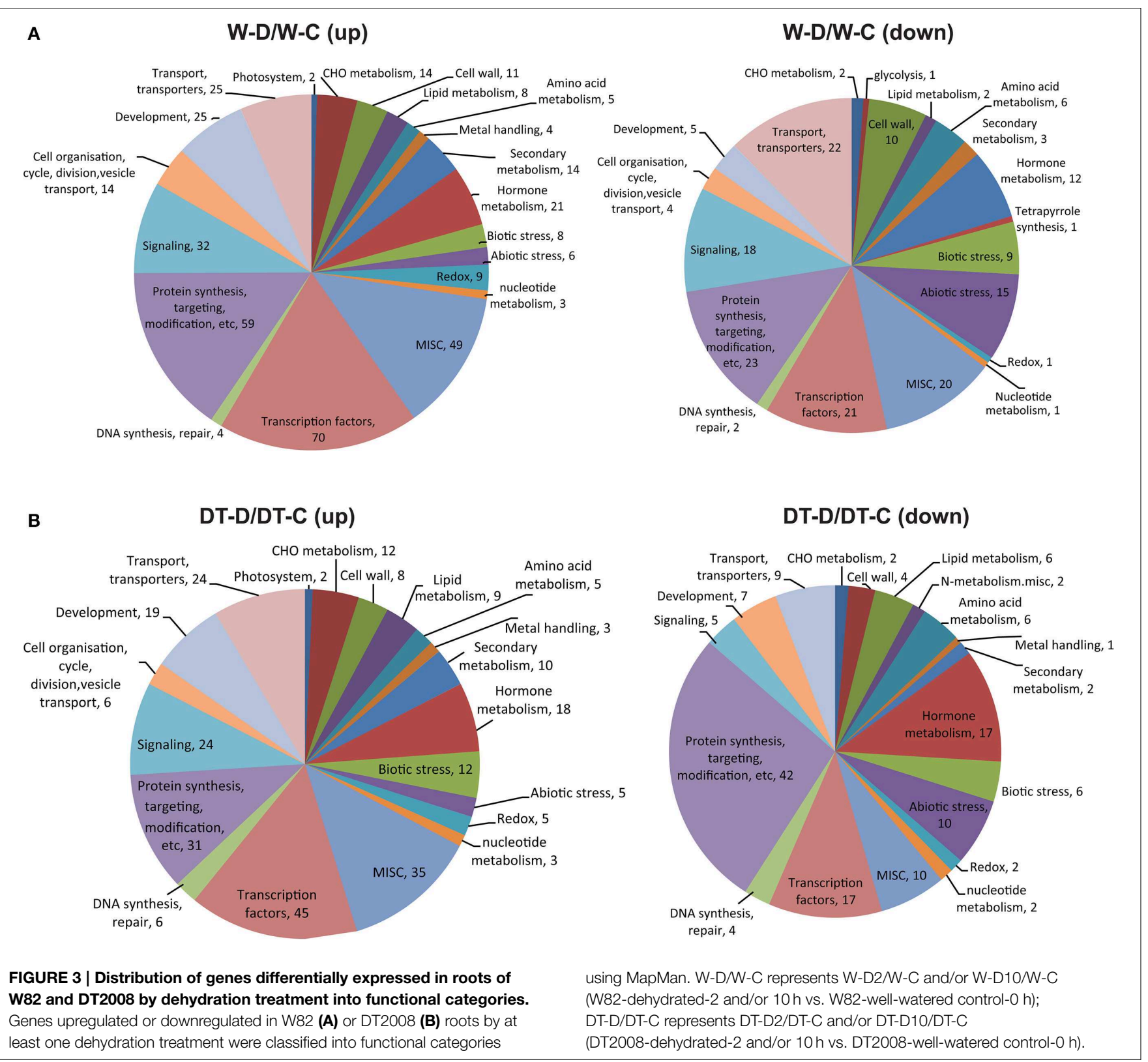

dehydration-repressible genes (W-D2/W-C, W-D10/W-C, DTD2/DT-C, and DT-D10/DT-C in Figure 4; Supplementary Figure S2; Supplementary Table S9). For instance, there were 13 and 5 upregulated GmNAC genes, in dehydrated W82 and DT2008 roots, respectively, while there were only 0 and 1 downregulated $G m N A C$ genes detected in the respective root samples (Supplementary Table S9). Another example is that among the AP2_EREBP-type members, 13 and 11 dehydrationinduced genes were found in W82 and DT2008 roots, respectively, in comparison with 3 and 4 dehydration-repressed genes in the respective dehydrated roots (Supplementary Table S9). Under our stringently set criteria of the fold change and $q$-values, the majority of the TF genes of these representative TF families were observed to be induced in either W82 or DT2008 roots by the prolonged $10 \mathrm{~h}$ rather than the short $2 \mathrm{~h}$ dehydration treatment (Figure 4; Supplementary Table S9).

Apart from the regulatory TFs, a number of DEGs encoding other types of regulatory proteins, such as kinases and hormone signaling-related proteins, were found in the signaling and protein modification categories. Some of them were predicted to be SnRK (sucrose non-fermenting-related), RLK (receptorlike), and MAP (mitogen-activated protein) kinases and PP2C (protein phosphatase 2C) proteins based on sequence homology with their Arabidopsis counterparts (Supplementary Figure S2; Supplementary Tables S6E,F, S7E,F). These proteins have been shown to be involved in regulation of plant responses to various stresses, including drought (Umezawa, 2011; Osakabe et al., 2013). Among many dehydration-inducible genes coding 


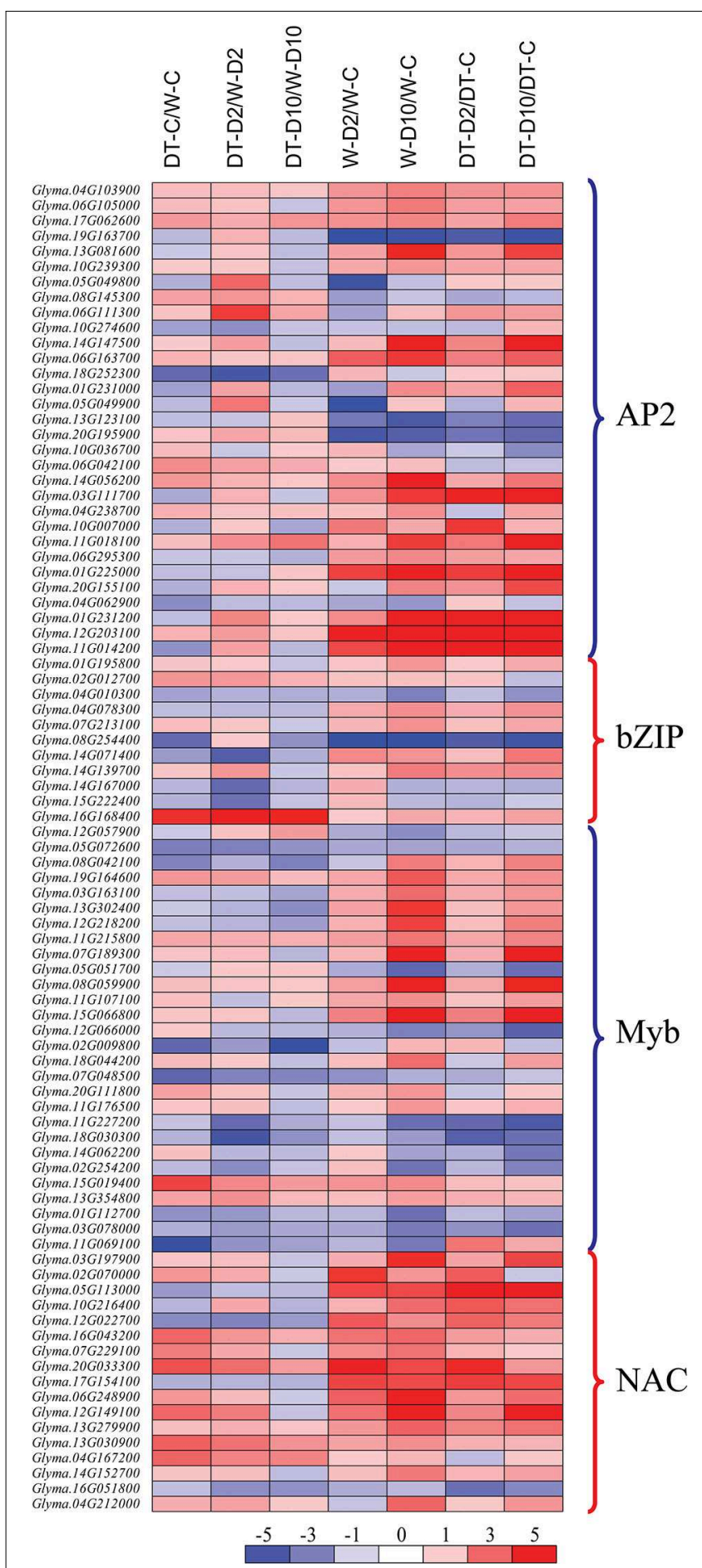

FIGURE 4 | Heatmap analysis of genes from well-known stress-related transcription factor families showing differential expression in various comparisons under well-watered and/or dehydration conditions.

DT-C/W-C, DT2008-well-watered control-0 h vs. W82-well-watered control-0 h; DT-D2/W-D2, DT2008-dehydrated-2 h vs. W82-dehydrated-2 h;

DT-D10/W-D10, DT2008-dehydrated-10 h vs. W82-dehydrated-10 h; W-D2/W-C, W82-dehydrated-2 h vs. W82-well-watered control-0 h; W-D10/W-C, W82-dehydrated-10 h vs. W82-well-watered control-0 h; DT-D2/DT-C, DT2008-dehydrated-2 h vs. DT2008-well-watered control-0 h; DT-D10/DT-C, DT2008-dehydrated-10 h vs. DT2008-well-watered control-0 h. for non-regulatory proteins are those encoding proteins of transporters, osmoprotectant biosynthesis-related proteins, and detoxification enzymes. Some are deserved to be mentioned, such as $\mathrm{ABC}$ (ATP-binding cassette) transporters, the ABA-importing transporter 1 (AIT1)-like proteins that might have ABA importer activity (Kanno et al., 2012), aquaporins, galactinol synthases, and polyamine oxidases (Supplementary Tables S6E, S7E). An appropriate change of their levels during stress may lead to a better adaptation of the plants (Osakabe et al., 2013; Himuro et al., 2014; Minocha et al., 2014; Rangan et al., 2014; Srivastava et al., 2014).

\section{Differential Expression between W82 and DT2008 Roots under Normal and Dehydration Conditions - the Upregulated Gene Sets}

To study the correlation between the differential gene expression in roots of W82 and DT2008 and their differential drought tolerance, we first compared their root transcriptomes under both normal and dehydration conditions. With regard to the upregulated gene sets derived from DT2008 vs. W82 comparison, we found that under well-watered conditions, 82 genes were upregulated in DT-C/W-C comparison, whereas under dehydration, a total of 147 genes were upregulated in DTD/W-D comparison, with more induced genes being identified during earlier stress (Figure 5A). Namely, 143 upregulated genes were found in DT-D2/W-D2, while only nine upregulated genes in DT-D10/W-D10 (Figure 5A; Supplementary Tables S10A-D). A number of upregulated genes identified in DT-C/W-C and DT-D/W-D comparisons possess putative regulatory functions, as they encode transcription factor, kinase and hormone-related proteins (Supplementary Figure S3).

Next, to identify genes that might contribute to higher drought-tolerant level of DT2008, we first searched for genes that are more highly expressed in drought-tolerant DT2008 than drought-sensitive W82 under normal conditions and are dehydration-inducible in W82 and/or DT2008 roots. We, therefore, subjected the upregulated gene sets obtained from the following comparisons DT-C/W-C, W-D/W-C, and DT-D/DT-C to a Venn analysis (DT-C/W-C vs. W-D/W-C, DT-C/W-C vs. DT-D/DT-C, DT-C/W-C vs. W-D/W-C vs. DT-D/DT-C) (Supplementary Tables S11A-C). As shown in Figure 5B, out of 82 genes displaying higher expression in DT2008 roots than in W82 roots (DT-C/W-C comparison, Supplementary Table S10A), six and two genes were found to be inducible by dehydration in W82 and DT2008 roots, respectively, with one gene, Glyma.04G083000, was upregulated in both dehydrated W82 and DT2008 roots (Supplementary Tables S11A-C). Furthermore, genes showing higher expression in DT2008 roots than in W82 roots under dehydration conditions, and being dehydration-inducible in W82 and/or DT2008 roots, might also have impact on improved drought-tolerant level of DT2008 vs. W82. Thus, the upregulated gene sets of DT-D/W-D, W-D/W$\mathrm{C}$, and DT-D/DT-C comparisons were also evaluated by a Venn analysis. Among 147 genes with more abundant transcripts in DT2008 roots than in W82 roots (DT-D/W-D, Supplementary Table S10D), 10 and 14 genes were detected to be upregulated in dehydrated W82 and DT2008 roots, respectively, of which five 


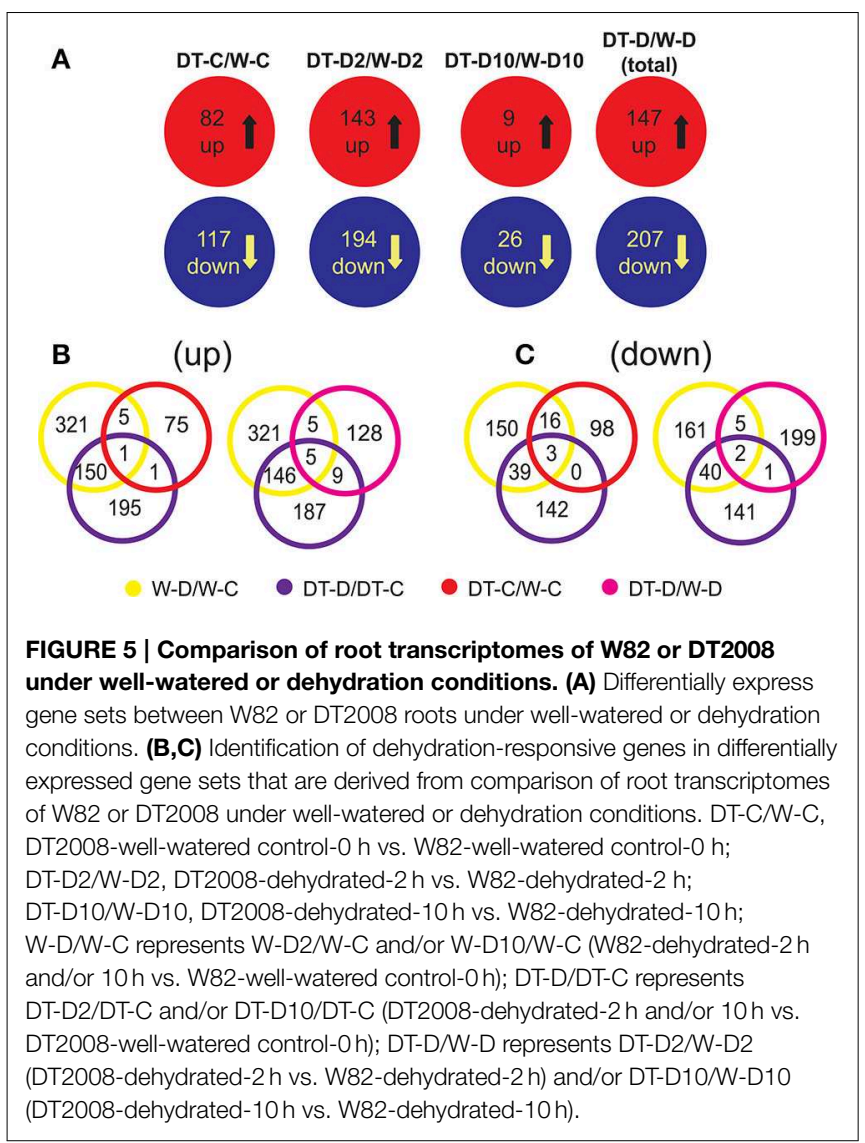

genes were upregulated in roots of both cultivars by dehydration (Figure 5B, Supplementary Tables S11D-F).

\section{Differential Expression between W82 and DT2008 Roots under Normal and Dehydration Conditions - the Downregulated Gene Sets}

As for the downregulated gene sets obtained from comparative analysis of W82 and DT2008 root transcriptomes, we detected 117 and 207 downregulated genes in DT-C/W-C (normal conditions) and DT-D/W-D (dehydration conditions) comparisons, respectively. We also observed that more genes (194 vs. 26 genes) were downregulated in DT-D/W-D comparison by the short-term $2 \mathrm{~h}$ (DT-D2/W-D2) rather than the prolonged $10 \mathrm{~h}$ (DT-D10/W-D10) dehydration treatment (Figure 5A; Supplementary Tables S10E-H).

In a similar manner, the downregulated gene sets obtained from the comparative analysis of root transcriptomes of W82 and DT2008 under non-stressed and stressed conditions (DT-C/WC and DT-D/W-D) were also analyzed to identify dehydrationrepressible genes exhibiting lower expression in drought-tolerant DT2008 as these genes would also be responsible for better performance of DT2008 relative to W82 under drought. Thus, downregulated gene sets of DT-C/W-C, DT-D/W-D, W-D/W$\mathrm{C}$, and DT-D/DT-C comparisons were evaluated by a Venn analysis as well (Supplementary Table S12). Venn diagrams shown in Figure 5C indicated that a total of 19 genes had lower expression in DT2008 roots than in W82 roots under well-watered conditions. All these 19 genes were repressed by dehydration in W82 roots, of which three genes were also downregulated in DT2008 roots (Supplementary Tables S12AC). As for genes showing lower expression levels in DT2008 roots than W82 roots under stress conditions, we found a total of eight genes of which five and one genes were repressed by dehydration in W82 or DT2008 roots only, while two genes were dehydrationrepressed in roots of both cultivars (Figure 5C, Supplementary Tables S12D-F).

\section{Discussion}

Large-scale transcriptome analysis is one of the most comprehensive approaches used to identify gene repertoire whose members are responsible to certain stressors (Mochida and Shinozaki, 2011). The completion of soybean genomic sequence has enabled us to carry out high-throughput transcriptomic studies in this important legume crop under various stress conditions in different organs (Schmutz et al., 2010; Le et al., 2012b; Ferreira Neto et al., 2013; Wan et al., 2015). Genes identified through the large-scale expression profiling studies, not only in soybean but also in other crops, have significantly accumulated in the past decade, providing a valuable resource for further functional genomics and comparative analyses ( $\mathrm{Ma}$ et al., 2012).

DT2008 is an elite soybean cultivar cultivated in many regions in Vietnam, owing to its strong tolerance to drought and dehydration in comparison with many other cultivars (Vinh et al., 2010; Ha et al., 2013; Sulieman et al., 2015). In a previous study, we compared the drought tolerability of DT2008 and the W82 model cultivar, and found that the higher drought-tolerant degree of DT2008 relative to W82 might be attributed, at least, to its better root development in comparison with W82 (Ha et al., 2013). To explain this phenomenon at molecular level, in the current study we carried out a microarray analysis of root transcriptomes of both DT2008 and W82 under normal, as well as mild ( $2 \mathrm{~h}$-treated) and severe ( $10 \mathrm{~h}$ treated) dehydration stress conditions using the $66 \mathrm{~K}$ soybean GeneChip (Figures 1A,B, Supplementary Table S1). This custom $66 \mathrm{~K}$ Affymetrix GeneChip has been shown to be a reliable tool for large-scale gene expression analysis in different organs under different types of stress, such as leaves (Le et al., 2012b) and roots (this work) under drought/dehydration stress, and in the same organs under biotic stress (Valdes-Lopez et al., 2011; Wan et al., 2015).

With the release of the newest annotation version Glyma v2.0 (http://phytozome.jgi.doe.gov/pz/portal.html), we were able to examine the expression of 38172 genes with high confidence through our transcriptome analysis (Supplementary Table S2). In general, we found more DEGs in roots of droughtsensitive W82 than in that of drought-tolerant DT2008 under dehydration in both upregulated and downregulated categories. Specifically, $2.15 \%$ (822/38172 genes) of the 38172 examined genes, which were annotated with high confidence, showed altered expression by dehydration in W82 roots, whereas $1.66 \%$ $(632 / 38172$ genes $)$ of the analyzed genes exhibited differential 
expression in DT2008 roots under the same treatment conditions (Supplementary Figure S1A). On the other hand, in another independent study using DeepSuperSAGE (26 bp tags) for comparative root transcriptome analysis of 15-day-old droughttolerant Embrapa 48 and drought-sensitive BR 16 seedlings at early stage of dehydration stress (between 0 and $150 \mathrm{~min}$ with 25 min interval), the authors in total found more differentially expressed soybean unitags in drought-tolerant Embrapa 48 roots than in drought-sensitive BR 16 roots in both upregulated and downregulated categories (Ferreira Neto et al., 2013). These findings suggest that different varieties might transcriptionally respond to dehydration/drought in different ways to activate root-related mechanisms for higher tolerability when compared with a specific drought-sensitive genotype. Alternatively, the different growth conditions might be a reason for the different observations of the two studies, as we grew the soybean plants in soil, whereas Ferreira Neto and colleagues hydroponically cultivated their soybean plants in nutrient solution (Ferreira Neto et al., 2013). It is worthy to notice that we also detected more upregulated genes in drought-tolerant DT2008 roots than drought-sensitive W82 roots by early $2 \mathrm{~h}$ dehydration treatment (131 vs. 105), although a reverse tendency was observed in case of downregulated genes (34 vs. 47) (Figure 1C, Supplementary Figure S1A). These results together suggest that induction of more dehydration/drought-responsive genes in roots of droughttolerant cultivars, as compared with that in drought-sensitive cultivar, at early stage of stress exposure might contribute to its higher drought tolerability (Vinh et al., 2010; Ferreira Neto et al., 2013; Ha et al., 2013).

In addition, we recorded more DEGs in roots of both DT2008 (DT-D10/DT-C vs. DT-D2/DT-C) and W82 (W-D10/W-C vs. $\mathrm{W}-\mathrm{D} 2 / \mathrm{W}-\mathrm{C}$ ) by $10 \mathrm{~h}$ than $2 \mathrm{~h}$ dehydration treatment (Figure 1C, Supplementary Figure S1A). These data indicated that the long-term dehydration stress triggered change in expression of more genes in soybean roots than the short-term one, independently of the genotype. Furthermore, the MAPMAN analysis showed that TF encoding genes were the most highly enriched upregulated genes, whereas those classified to "protein synthesis, targeting, modification, etc" category were the most highly enriched downregulated genes in both W82 and DT2008 roots under dehydration (Figure 3). This finding suggested that genes belonging to these categories were those whose expression in roots is the most responsive to dehydration to aid the plants in adapting to the stress. Interestingly, a previous microarray analysis using the same GeneChip found a reverse trend in V6 and R2 leaves of the W82 cultivar. The authors reported that in these W82 leaf tissues, TF encoding genes were enriched among the downregulated genes; while, for the upregulated gene sets, "protein synthesis, targeting, modification, etc" was the most significantly enriched category (Le et al., 2012b).

With respect to the TF encoding genes, many members of the major TF families, such as AP2_EREBP, bZIP, MYB, and NAC, showed differential expression by dehydration in both W82 and DT2008 roots (Figure 4). Moreover, the heatmap analysis also indicated that the majority of the dehydration-inducible TF genes, such as NAC genes, exhibited higher expression level in
DT2008 roots than W82 roots, especially under well-watered and early dehydration treatment (Figure 4). Increasing evidence has shown that members of these TF families play important roles in plant responses to water deficit by controlling transcription of downstream genes through their specific binding to the socalled cis-acting elements located in the promoters of target genes (Yamaguchi-Shinozaki and Shinozaki, 2006; Hadiarto and Tran, 2011; Jogaiah et al., 2013). A number of published reports have shown positive correlation between NAC gene expression levels, specifically in roots or leaves or whole plants, and drought tolerability of various crops, including soybean (Nakashima et al., 2007; Zheng et al., 2009; Xue et al., 2011; Thao et al., 2013; Thu et al., 2014; Zhu et al., 2014; Nguyen et al., 2015; Yang et al., 2015), further supporting that NAC TFs, at least in part, might contribute to the higher drought tolerance of DT2008 vs. W82. Molecular tailoring of the TF encoding genes has provided a promising approach for improvement of tolerance of a number of crops to various types of environmental stresses, including drought (Yang et al., 2010; Thao and Tran, 2012).

Apart from the TF genes, many other dehydration-inducible genes also displayed higher expression levels in DT2008 roots than W82 roots under normal or dehydration conditions (Supplementary Table S11), which might contribute to differential drought tolerance of DT2008 and W82. Results summarized in Figure 5 indicated that the short-term dehydration-induced expression changes might be more highly required for enhanced drought tolerance of DT2008 vs. W82 than the prolonged dehydration-induced ones, as significantly higher number of DEGs were identified in DTD2/WT-D2 comparison than DT-D10/WT-D10 comparison. With regard to dehydration-upregulated genes with higher expression levels in DT2008 roots vs. W82 roots under well-watered conditions (Supplementary Tables S11A-C), Glyma.14G216500 encodes an ortholog STH2 (salt tolerance homolog2) (Table 1), a B-box TF that can act as a positive regulator of photomorphogenesis and anthocyanin biosynthesis (Datta et al., 2007). This gene might play a role in enhanced tolerance of DT2008 as anthocyanins are known to protect plants against various environmental stresses, including drought (Pourcel et al., 2007). Modulation of the signaling molecule phospholipids, which involved in regulation of plant response to environmental stimuli, through Glyma.04G083000 (induced by dehydration in both DT2008 and W82 background, Table 1) and Glyma.20G189100 that encode putative proteins with function in phosphoinositide signaling, might also be responsible for increased tolerance of DT2008 to drought (Liu et al., 2013). As for the dehydration-inducible genes showing higher transcription levels in DT2008 roots vs. W82 roots under dehydration (Supplementary Tables S11D-F), Glyma.19G227800, Glyma.16G003500, and Glyma.13G095200 encoding osmoprotectant biosynthesis-, detoxification- or cell wall-related proteins (Table 1), such as the orthologs of Arabidopsis AtGOLS2 (Arabidopsis thaliana galactinol synthase 2), glyoxalase I family protein and xyloglucan endotransglycosylase, may play important roles in better adaptation of DT2008 to drought relative to W82 as supported 
TABLE 1 | List of several candidate genes that might contribute to higher drought tolerance of DT2008 vs. W82.

\begin{tabular}{|c|c|c|c|c|c|c|c|}
\hline \multirow[t]{2}{*}{ Glyma 2 ID } & \multirow{2}{*}{$\begin{array}{l}\text { Arabidopsis } \\
\text { ortholog }\end{array}$} & \multirow[t]{2}{*}{ Description } & \multirow{2}{*}{$\frac{\text { W-D/W-C }}{\text { Responsiveness }}$} & \multirow{2}{*}{$\begin{array}{l}\text { DT-D/DT-C } \\
\text { Responsiveness }\end{array}$} & \multirow{2}{*}{$\begin{array}{c}\text { DT-C/W-C } \\
\text { Fold change } \\
(q<0.05)\end{array}$} & \multirow{2}{*}{$\begin{array}{c}\text { DT-D2/W-D2 } \\
\text { Fold change } \\
(q<0.05)\end{array}$} & \multirow{2}{*}{$\begin{array}{c}\text { DT-D10/W-D10 } \\
\text { Fold change } \\
(q<0.05)\end{array}$} \\
\hline & & & & & & & \\
\hline Glyma.04G083000 & AT3G22810 & $\begin{array}{l}\text { Putative phosphoinositide binding } \\
\text { protein }\end{array}$ & Induced & Induced & 2.61 & Unchanged & Unchanged \\
\hline Glyma.20G189100 & AT3G10550 & $\begin{array}{l}\text { Putative inositol or } \\
\text { phosphatidylinositol phosphatase }\end{array}$ & Induced & None & 2.03 & Unchanged & Unchanged \\
\hline Glyma.14G216500 & AT1G75540 & $\begin{array}{l}\text { Similar to STH2 (salt tolerance } \\
\text { homolog2) }\end{array}$ & Induced & None & 4.40 & Unchanged & Unchanged \\
\hline Glyma. 13G095200 & AT4G25810 & $\begin{array}{l}\text { Similar to XTR6 (xyloglucan } \\
\text { endotransglycosylase 6) }\end{array}$ & None & Induced & Unchanged & 2.16 & Unchanged \\
\hline Glyma.16G003500 & AT1G80160 & Similar to GLYI7 (glyoxylase I 7) & Induced & None & Unchanged & 2.91 & Unchanged \\
\hline Glyma.19G227800 & AT1G56600 & $\begin{array}{l}\text { Similar to AtGOLS2 (A.thaliana } \\
\text { galactinol synthase 2) }\end{array}$ & Induced & Induced & Unchanged & 3.70 & Unchanged \\
\hline Glyma.02G069400 & AT4G18710 & $\begin{array}{l}\text { Similar to BIN2 } \\
\text { (brassinosteroid-insensitive 2) }\end{array}$ & Repressed & Repressed & -3.56 & Unchanged & Unchanged \\
\hline Glyma.06G050900 & AT5G36250 & Similar to protein phosphatase $2 \mathrm{C} 74$ & Repressed & Repressed & -3.23 & -2.30 & Unchanged \\
\hline Glyma.08G254400 & АT3G30530 & Similar to bZIP42 & Repressed & Repressed & Unchanged & Unchanged & -2.05 \\
\hline Glyma.12G202400 & AT4G03415 & Similar to protein phosphatase 2C 52 & Repressed & None & -2.11 & Unchanged & Unchanged \\
\hline
\end{tabular}

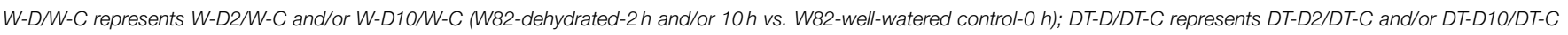

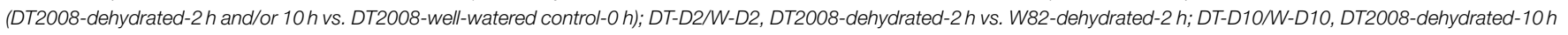
vs. W82-dehydrated-10h.

by previous studies (Xu et al., 1995; Taji et al., 2002; Kaur et al., 2014).

Additionally, dehydration-repressible genes with lower expression level in drought-tolerant DT2008 roots than droughtsensitive W82 might also contribute to the better performance of DT2008 vs. W82 under drought. Among the dehydrationrepressible genes that had lower expression levels in DT2008 than W82 under well-watered conditions (Supplementary Tables S12A-C), Glyma.02G069400 codes for an ortholog of Arabidopsis BIN2 (brassinosteroid-insensitive 2) (Table 1). It was reported that the rice ortholog of BIN2, the OsGSK1 (glycogen synthase 3 -like protein kinase), acts as a negative regulator of plant responses to multiple stresses, including drought (Koh et al., 2007). Repression of Glyma.02G069400 might therefore contribute to increased drought tolerance of DT2008. Another example is Glyma.12G202400 encoding a protein with high homology to an Arabidopsis phosphatase $2 \mathrm{C}$ protein (AT4G03415) (Table 1) that might be involved in drought response perhaps through its interaction with CPK16 of $\mathrm{Ca}^{2+}$-dependent protein kinase/sucrose non-fermenting related kinase (CPK/SnRK) superfamily (Curran et al., 2011). CPK16 has been known to be implicated in regulation of root gravitropism that is a trait important for plant response to water stress (Kirkham, 2008; Huang et al., 2013). With regard to the dehydration-repressible genes that displayed lower expression levels in DT2008 roots than W82 roots under dehydration (Figure 5C; Supplementary Tables S12D-F), Glyma.06G050900 and Glyma.08G254400 encoding phosphatase 2C and bZIP orthologs of Arabidopsis, respectively, caught our attention (Table 1). Glyma.06G050900 exhibited lower expression in DT2008 roots than W82 roots under both normal and dehydration conditions (Table 1). It is well established that many members of the phosphatase $2 \mathrm{C}$ family are involved in stress signaling (Schweighofer et al., 2004). Several phosphatase $2 \mathrm{C}$ proteins have been found to act as negative regulators of ABA signaling (Umezawa et al., 2010). As for Glyma.08G254400, although there is no specific information either for this gene or its highest Arabidopsis homolog (AT3G30530/AtbZIP42), there have been published reports that several bZIP TFs act as negative regulators of drought tolerance. For instance, overexpression of OsbZIP52 in rice significantly enhanced sensitivity of transgenic plants to cold and drought stresses (Liu et al., 2012). Thus, downregulation of such a gene would allow plants to adapt better to adverse environmental conditions.

In summary, our comparative analysis of root transcriptomes of DT2008 and W82 under both well-watered and dehydration conditions have allowed us to identify genetic components that might contribute to the improved drought tolerance of DT2008. Our study also provides a useful genetic resource for scientists with interests in basic and/or applied research to carry out further in-depth gene characterization and functional analyses. This in turn will contribute to deeper understanding of mechanisms regulating drought responses and adaptation in soybean, which ultimately leads to development of soybean cultivars with improved tolerance to drought.

\section{Materials and Methods}

\section{Plant Growth and Dehydration Treatment}

W82 and DT2008 soybean plants were separately grown in pots containing vermiculite (6 plants per 6-liter pot) under 
well-watered conditions in a controlled greenhouse (continuous $30^{\circ} \mathrm{C}$ temperature, photoperiod of $12 / 12 \mathrm{~h}, 150 \mu \mathrm{mol} \mathrm{m}^{-2} \mathrm{~s}^{-1}$ photon flux density). For the collection of well-watered and dehydrated root tissues, 14-d-old soybean plants with two trifoliate leaves (V2 stage) were carefully removed from pots, then gently washed to remove soil from the roots. Subsequently, the W82 and DT2008 plants were dried on a filter paper for different time periods under the condition of $44 \%$ relative humidity, $23^{\circ} \mathrm{C}$ room temperature and $10 \mu \mathrm{mol} \mathrm{m} \mathrm{m}^{-2} \mathrm{~s}^{-1}$ photon flux light intensity. The severity of the stress level was measured by determination of RWC of the aerial parts of dehydrated plants. After the dehydration treatment, plants dehydrated for 0,2 , and $10 \mathrm{~h}$ were collected and the roots were separated from the shoots. Root samples were quickly frozen in liquid nitrogen and stored at $-80^{\circ} \mathrm{C}$ until RNA purification. Accordingly, the following root samples were collected in three biological replicates from W82 and DT2008 plants for microarray analysis: W82 wellwatered control $0 \mathrm{~h}(\mathrm{~W}-\mathrm{C}), \mathrm{W} 82$ dehydrated $2 \mathrm{~h}$ (W-D2), W82 dehydrated $10 \mathrm{~h}$ (W-D10), DT2008 well-watered control $0 \mathrm{~h}$ (DTC), DT2008 dehydrated $2 \mathrm{~h}$ (DT-D2); DT2008 dehydrated $10 \mathrm{~h}$ (DT-D10).

\section{Microarray Analysis of the Root Samples using 61K Affymetrix Microarray}

RNAs were extracted from root samples using the Trizol reagent (Invitrogen, Carlsbad, CA, USA) as recommended by the manufacturer's protocol. Purified total RNA was subsequently subjected to a DNase I treatment prior to the quality assessment by an Agilent 2100 Bioanalyzer (Le et al., 2011a). For microarray analysis, cDNA synthesis, cRNA amplification, and synthesis of sense strand cDNAs were carried out using the Ambion WT expression kit. cDNA labeling was carried out using Affymetrix GeneChip WT Terminal Labeling Kit according to the supplier's instructions. Hybridization and scanning of hybridized arrays (G2505B microarray scanner, Agilent Technologies) were performed as described previously (Nishiyama et al., 2012). Three biological replicates collected from each treatment were subjected to the microarray experiment. Microarray data were analyzed using Affymetrix Expression Console with library supplied from Affymetrix and GeneSpring (Ver. 11) as essentially described (Le et al., 2012b). Statistical significance of each gene in each treatment ( $p$-value) was estimated by a Student's $t$-test, while its certainty level (the corrected $p$-values, i.e., $q$-values) was assessed using Benjamini and Hochberg False Discovery Rate. Genes with expression change $\geq 2$-fold $(q<0.05)$ were regarded to be differentially expressed. The obtained microarray data have been deposited in the Gene Expression Omnibus (GEO) database (http://www.

\section{References}

Choudhary, S. P., and Tran, L. S. (2011). Phytosterols: perspectives in human nutrition and clinical therapy. Curr. Med. Chem. 18, 4557-4567. doi: 10.2174/092986711797287593

Comas, L. H., Becker, S. R., Cruz, V. M., Byrne, P. F., and Dierig, D. A. (2013). Root traits contributing to plant productivity ncbi.nlm.nih.gov/geo/browse/?view=series) (accession number GSE65553) ${ }^{1}$.

\section{MapMan Analysis of the Root Transcriptomes}

MapMan (http://mapman.gabipd.org) was used to annotate and analyze the microarray data as according to previously published methods (Thimm et al., 2004; Le et al., 2012b; Nishiyama et al., 2012; Ha et al., 2014). The lists containing DEGs obtained from corresponding comparisons were supplied to MapMan for classification of DEGs into functional groups.

\section{Validation of Microarray Data by RT-qPCR}

Several genes were randomly selected for verification of the microarray data using RT-qPCR.

The specific primer pairs used in RT-qPCR were listed in Supplementary Table S8. The Fbox gene was used as a reference gene in the RT-qPCR analysis of RNA samples from three biological replicates (Le et al., 2012a). Preparation of cDNAs from DNase I-treated RNA samples for RT-qPCR was performed as previously described (Le et al., 2011b).

\section{Author Contributions}

L-SPT conceived research and wrote the manuscript. $\mathrm{CVH}$, YW, UTT, DTL, MT, and KHN performed the experiments and analyzed the data. MS and DVN contributed research materials.

\section{Acknowledgments}

$\mathrm{CVH}$ and $\mathrm{KHN}$ appreciate the support from International Program Associate of RIKEN for their PhD study. This work was supported in part by a grant (Project Code 03/2012/HĐĐTĐL) from the Vietnam Ministry of Science and Technology to the Research Group of DVN, and by a grant from Japan Science and Technology Agency (JST), Core Research for Evolutionary Science and Technology (CREST) to MS. The authors declare no conflict of interest.

\section{Supplementary Material}

The Supplementary Material for this article can be found online at: http://journal.frontiersin.org/article/10.3389/fpls.2015. 00551

\footnotetext{
${ }^{1}$ Accession number to microarray data deposited at Gene Expression Omnibus database: GSE65553.
} 
Datta, S., Hettiarachchi, C., Johansson, H., and Holm, M. (2007). SALT TOLERANCE HOMOLOG2, a B-Box protein in Arabidopsis that activates transcription and positively regulates light-mediated development. Plant Cell 19, 3242-3255. doi: 10.1105/tpc.107.054791

Deshmukh, R., Sonah, H., Patil, G., Chen, W., Prince, S., Mutava, R., et al. (2014). Integrating omic approaches for abiotic stress tolerance in soybean. Front. Plant Sci. 5:244. doi: 10.3389/fpls.2014.00244

Ferreira Neto, J. R., Pandolfi, V., Guimaraes, F. C., Benko-Iseppon, A. M., Romero, C., Silva, R. L., et al. (2013). Early transcriptional response of soybean contrasting accessions to root dehydration. PLoS ONE 8:e83466. doi: 10.1371/journal.pone.0083466

Ha, C. V., Le, D. T., Nishiyama, R., Watanabe, Y., Tran, U. T., Dong, N. V., et al. (2013). Characterization of the newly developed soybean cultivar DT2008 in relation to the model variety W82 reveals a new genetic resource for comparative and functional genomics for improved drought tolerance. Biomed Res. Int. 2013:759657. doi: 10.1155/2013/759657

Ha, C. V., Leyva-Gonzalez, M. A., Osakabe, Y., Tran, U. T., Nishiyama, R., Watanabe, Y., et al. (2014). Positive regulatory role of strigolactone in plant responses to drought and salt stress. Proc. Natl. Acad. Sci. U.S.A. 111, 851-856. doi: 10.1073/pnas.1322135111

Ha, S., Vankova, R., Yamaguchi-Shinozaki, K., Shinozaki, K., and Tran, L. S. (2012). Cytokinins: metabolism and function in plant adaptation to environmental stresses. Trends Plant Sci. 17, 172-179. doi: 10.1016/j.tplants.2011.12.005

Hadiarto, T., and Tran, L. S. (2011). Progress studies of drought-responsive genes in rice. Plant Cell Rep. 30, 297-310. doi: 10.1007/s00299-010-0956-Z

Himuro, Y., Ishiyama, K., Mori, F., Gondo, T., Takahashi, F., Shinozaki, K., et al. (2014). Arabidopsis galactinol synthase AtGolS2 improves drought tolerance in the monocot model Brachypodium distachyon. J. Plant Physiol. 171, 1127-1131. doi: 10.1016/j.jplph.2014.04.007

Hossain, Z., Khatoon, A., and Komatsu, S. (2013). Soybean proteomics for unraveling abiotic stress response mechanism. J. Proteome Res. 12, 4670-4684. doi: $10.1021 /$ pr400604b

Hsien, L. Y. (2015). "Utilization of vegetable oil as bio-lubricant and additive," in Towards Green Lubrication in Machining, ed L. Y. Hsien (New York, NY: Springer), 7-17.

Huang, S. J., Chang, C. L., Wang, P. H., Tsai, M. C., Hsu, P. H., and Chang, I. F. (2013). A type III ACC synthase, ACS7, is involved in root gravitropism in Arabidopsis thaliana. J. Exp. Bot. 64, 4343-4360. doi: 10.1093/jxb/ert241

Jogaiah, S., Ramsandra Govind, S., and Tran, L. S. (2013). System biology-based approaches towards understanding drought tolerance in food crops. Crit. Rev. Biotechnol. 33, 23-39. doi: 10.3109/07388551.2012.659174

Kanno, Y., Hanada, A., Chiba, Y., Ichikawa, T., Nakazawa, M., Matsui, M., et al. (2012). Identification of an abscisic acid transporter by functional screening using the receptor complex as a sensor. Proc. Natl. Acad. Sci. U.S.A. 109, 9653-9658. doi: 10.1073/pnas. 1203567109

Karan, R., and Subudhi, P. K. (2014). Overexpression of an adenosine diphosphateribosylation factor gene from the halophytic grass Spartina alterniflora confers salinity and drought tolerance in transgenic Arabidopsis. Plant Cell Rep. 33, 373-384. doi: 10.1007/s00299-013-1537-8

Kaur, C., Ghosh, A., Pareek, A., Sopory, S. K., and Singla-Pareek, S. L. (2014). Glyoxalases and stress tolerance in plants. Biochem. Soc. Trans. 42, 485-490. doi: 10.1042/BST20130242

Khan, A., Garbelli, A., Grossi, S., Florentin, A., Batelli, G., Acuna, T., et al. (2014). The Arabidopsis STRESS RESPONSE SUPPRESSOR DEAD-box RNA helicases are nucleolar- and chromocenter-localized proteins that undergo stress-mediated relocalization and are involved in epigenetic gene silencing. Plant J. 79, 28-43. doi: 10.1111/tpj.12533

Kirkham, M. B. (2008). Horizontal root growth: water uptake and stomatal resistance under microgravity. Vadose Zone J. 7, 1125-1131. doi: 10.2136/vzj2007.0097

Koh, S., Lee, S. C., Kim, M. K., Koh, J. H., Lee, S., An, G., et al. (2007). TDNA tagged knockout mutation of rice OsGSK1, an orthologue of Arabidopsis BIN2, with enhanced tolerance to various abiotic stresses. Plant Mol. Biol. 65, 453-466. doi: 10.1007/s11103-007-9213-4

Ku, Y.-S., Au-Yeung, W.-K., Yung, Y.-L., Li, M.-W., Wen, C.-Q., Liu, X., et al. (2013). "Drought stress and tolerance in soybean," in A Comprehensive Survey of Internaitonal Soybean Research - Genetics, Physiology, Agronomy and Nitrogen Relationships, ed J. E. Board (New York, NY: InTech), 209-237.
Le, D. T., Aldrich, D. L., Valliyodan, B., Watanabe, Y., Ha, C. V., Nishiyama, R., et al. (2012a). Evaluation of candidate reference genes for normalization of quantitative RT-PCR in soybean tissues under various abiotic stress conditions. PLoS ONE 7:e46487. doi: 10.1371/journal.pone.00 46487

Le, D. T., Nishiyama, R., Watanabe, Y., Mochida, K., Yamaguchi-Shinozaki, K., Shinozaki, K., et al. (2011a). Genome-wide expression profiling of soybean twocomponent system genes in soybean root and shoot tissues under dehydration stress. DNA Res. 18, 17-29. doi: 10.1093/dnares/dsq032

Le, D. T., Nishiyama, R., Watanabe, Y., Mochida, K., Yamaguchi-Shinozaki, K., Shinozaki, K., et al. (2011b). Genome-wide survey and expression analysis of the plant-specific NAC transcription factor family in soybean during development and dehydration stress. DNA Res. 18, 263-276. doi: 10.1093/dnares/dsr015

Le, D. T., Nishiyama, R., Watanabe, Y., Tanaka, M., Seki, M., Ham, L. H., et al. (2012b). Differential gene expression in soybean leaf tissues at late developmental stages under drought stress revealed by genome-wide transcriptome analysis. PLoS ONE 7:e49522. doi: 10.1371/journal.pone.0049522

Liu, C. T., Wu, Y. B., and Wang, X. P. (2012). bZIP transcription factor OsbZIP52/RISBZ5: a potential negative regulator of cold and drought stress response in rice. Planta 235, 1157-1169. doi: 10.1007/s00425-01 1-1564-Z

Liu, X., Zhai, S., Zhao, Y., Sun, B., Liu, C., Yang, A., et al. (2013). Overexpression of the phosphatidylinositol synthase gene $(Z m P I S)$ conferring drought stress tolerance by altering membrane lipid composition and increasing $\mathrm{ABA}$ synthesis in maize. Plant Cell Environ. 36, 1037-1055. doi: 10.1111/pce. 12040

Ma, Y., Qin, F., and Tran, L. S. (2012). Contribution of genomics to gene discovery in plant abiotic stress responses. Mol. Plant 5, 1176-1178. doi: $10.1093 / \mathrm{mp} / \mathrm{sss} 085$

Manavalan, L. P., Guttikonda, S. K., Tran, L. S., and Nguyen, H. T. (2009). Physiological and molecular approaches to improve drought resistance in soybean. Plant Cell Physiol. 50, 1260-1276. doi: 10.1093/pcp/pcp082

Minocha, R., Majumdar, R., and Minocha, S. C. (2014). Polyamines and abiotic stress in plants: a complex relationship. Front. Plant Sci. 5:175. doi: $10.3389 /$ fpls.2014.00175

Mochida, K., and Shinozaki, K. (2011). Advances in omics and bioinformatics tools for systems analyses of plant functions. Plant Cell Physiol. 52, 2017-2038. doi: $10.1093 / \mathrm{pcp} / \mathrm{pcr} 153$

Nakashima, K., Tran, L. S., Van Nguyen, D., Fujita, M., Maruyama, K., Todaka, D., et al. (2007). Functional analysis of a NAC-type transcription factor OsNAC6 involved in abiotic and biotic stress-responsive gene expression in rice. Plant J. 51, 617-630. doi: 10.1111/j.1365-313X.2007.03168.x

Nguyen, K. H., Ha, C. V., Watanabe, Y., Tran, U. T., Nasr Esfahani, M., Nguyen, D. V., et al. (2015). Correlation between differential drought tolerability of two contrasting drought-responsive chickpea cultivars and differential expression of a subset of CaNAC genes under normal and dehydration conditions. Front. Plant Sci. 6:449. doi: 10.3389/fpls.2015.00449

Nishiyama, R., Le, D. T., Watanabe, Y., Matsui, A., Tanaka, M., Seki, M., et al. (2012). Transcriptome analyses of a salt-tolerant cytokinin-deficient mutant reveal differential regulation of salt stress response by cytokinin deficiency. PLoS ONE 7:e32124. doi: 10.1371/journal.pone.0032124

Osakabe, Y., Yamaguchi-Shinozaki, K., Shinozaki, K., and Tran, L. S. (2013). Sensing the environment: key roles of membrane-localized kinases in plant perception and response to abiotic stress. J. Exp. Bot. 64, 445-458. doi: $10.1093 /$ jxb/ers354

Pourcel, L., Routaboul, J. M., Cheynier, V., Lepiniec, L., and Debeaujon, I. (2007). Flavonoid oxidation in plants: from biochemical properties to physiological functions. Trends Plant Sci. 12, 29-36. doi: 10.1016/j.tplants.2006. 11.006

Rangan, P., Subramani, R., Kumar, R., Singh, A. K., and Singh, R. (2014). Recent advances in polyamine metabolism and abiotic stress tolerance. Biomed Res. Int. 2014:239621. doi: 10.1155/2014/239621

Sadeghipour, O., and Abbasi, S. (2012). Soybean response to drought and seed inoculation. World Appl. Sci. J. 17, 55-60. Available online at: http://www.idosi.org/wasj/wasj17\%281\%2912/8.pdf

Satbhai, S. B., Ristova, D., and Busch, W. (2015). Underground tuning: quantitative regulation of root growth. J. Exp. Bot. 66, 1099-1112. doi: 10.1093/jxb/eru529 
Schmutz, J., Cannon, S. B., Schlueter, J., Ma, J., Mitros, T., Nelson, W., et al. (2010). Genome sequence of the palaeopolyploid soybean. Nature 463, 178-183. doi: 10.1038 /nature 08670

Schweighofer, A., Hirt, H., and Meskiene, I. (2004). Plant PP2C phosphatases: emerging functions in stress signaling. Trends Plant Sci. 9, 236-243. doi: 10.1016/j.tplants.2004.03.007

Srivastava, A. K., Penna, S., Nguyen, D. V., and Tran, L. S. (2014). Multifaceted roles of aquaporins as molecular conduits in plant responses to abiotic stresses. Crit. Rev. Biotechnol. doi: 10.3109/07388551.2014. 973367. [Epub ahead of print].

Sulieman, S., Van Ha, C., Nasr Esfahani, M., Watanabe, Y., Nishiyama, R., Pham, C. T., et al. (2015). DT2008: a promising new genetic resource for improved drought tolerance in soybean when solely dependent on symbiotic $\mathrm{N}_{2}$ fixation. Biomed Res. Int. 2015:687213. doi: 10.1155/2015/6 87213

Taji, T., Ohsumi, C., Iuchi, S., Seki, M., Kasuga, M., Kobayashi, M., et al. (2002). Important roles of drought- and cold-inducible genes for galactinol synthase in stress tolerance in Arabidopsis thaliana. Plant J. 29, 417-426. doi: 10.1046/j.0960-7412.2001.01227.x

Thao, N. P., and Tran, L. S. (2012). Potentials toward genetic engineering of drought-tolerant soybean. Crit. Rev. Biotechnol. 32, 349-362. doi: 10.3109/07388551.2011.643463

Thao, N. P., Thu, N. B., Hoang, X. L., Van Ha, C., and Tran, L. S. (2013). Differential expression analysis of a subset of drought-responsive GmNAC genes in two soybean cultivars differing in drought tolerance. Int. J. Mol. Sci. 14, 23828-23841. doi: 10.3390/ijms141223828

Thimm, O., Blasing, O., Gibon, Y., Nagel, A., Meyer, S., Kruger, P., et al. (2004). MAPMAN: a user-driven tool to display genomics data sets onto diagrams of metabolic pathways and other biological processes. Plant J. 37, 914-939. doi: 10.1111/j.1365-313X.2004.02016.X

Thu, N. B., Hoang, X. L., Doan, H., Nguyen, T. H., Bui, D., Thao, N. P., et al. (2014). Differential expression analysis of a subset of GmNAC genes in shoots of two contrasting drought-responsive soybean cultivars DT51 and MTD720 under normal and drought conditions. Mol. Biol. Rep. 41, 5563-5569. doi: 10.1007/s11033-014-3507-9

Tran, L. S., and Mochida, K. (2010). Functional genomics of soybean for improvement of productivity in adverse conditions. Funct. Integr. Genomics 10, 447-462. doi: 10.1007/s10142-010-0178-Z

Tran, L. S., and Nguyen, H. T. (2009). "Future biotechnology of legumes," in Nitrogen Fixation in Crop Production, eds D. W. Emerich and H. B. Krishnan (Madison, WI: American Society of Agronomy, Crop Science Society of America, Soil Science Society of America), 265-308.

Umezawa, T. (2011). Systems biology approaches to abscisic acid signaling. J. Plant Res. 124, 539-548. doi: 10.1007/s10265-011-0418-x

Umezawa, T., Nakashima, K., Miyakawa, T., Kuromori, T., Tanokura, M., Shinozaki, K., et al. (2010). Molecular basis of the core regulatory network in ABA responses: sensing, signaling and transport. Plant Cell Physiol. 51, 1821-1839. doi: $10.1093 /$ pcp/pcq156
Valdes-Lopez, O., Thibivilliers, S., Qiu, J., Xu, W. W., Nguyen, T. H., Libault, M., et al. (2011). Identification of quantitative trait loci controlling gene expression during the innate immunity response of soybean. Plant Physiol. 157, 1975-1986. doi: 10.1104/pp.111.183327

Vinh, M. Q., Chung, P. T. B., Manh, N. V., and Hong, L. T. A. (2010). Results of research, creation, drought-tolerant soybean variety, DT2008. J. Vietnamese Agric. Sci. Tech 6, 46-50. doi: 10.1007/s10142-010-0178-z

Wan, J., Vuong, T., Jiao, Y., Joshi, T., Zhang, H., Xu, D., et al. (2015). Wholegenome gene expression profiling revealed genes and pathways potentially involved in regulating interactions of soybean with cyst nematode (Heterodera glycines Ichinohe). BMC Genomics 16:148. doi: 10.1186/s12864-015-1316-8

Xu, W., Purugganan, M. M., Polisensky, D. H., Antosiewicz, D. M., Fry, S. C., and Braam, J. (1995). Arabidopsis TCH4, regulated by hormones and the environment, encodes a xyloglucan endotransglycosylase. Plant Cell 7, 1555-1567. doi: 10.1105/tpc.7.10.1555

Xue, G. P., Way, H. M., Richardson, T., Drenth, J., Joyce, P. A., and McIntyre, C. L. (2011). Overexpression of TaNAC69 leads to enhanced transcript levels of stress up-regulated genes and dehydration tolerance in bread wheat. Mol. Plant 4, 697-712. doi: $10.1093 / \mathrm{mp} / \mathrm{ssr} 013$

Yamaguchi-Shinozaki, K., and Shinozaki, K. (2006). Transcriptional regulatory networks in cellular responses and tolerance to dehydration and cold stresses. Annu. Rev. Plant Biol. 57, 781-803. doi: 10.1146/annurev.arplant.57.032905. 105444

Yang, S., Vanderbeld, B., Wan, J., and Huang, Y. (2010). Narrowing down the targets: towards successful genetic engineering of drought-tolerant crops. Mol. Plant 3, 469-490. doi: 10.1093/mp/ssq016

Yang, X., Wang, X., Ji, L., Yi, Z., Fu, C., Ran, J., et al. (2015). Overexpression of a Miscanthus lutarioriparius NAC gene MlNAC5 confers enhanced drought and cold tolerance in Arabidopsis. Plant Cell Rep. 34, 943-958. doi: 10.1007/s00299015-1756-2

Zheng, X., Chen, B., Lu, G., and Han, B. (2009). Overexpression of a NAC transcription factor enhances rice drought and salt tolerance. Biochem. Biophys. Res. Commun. 379, 985-989. doi: 10.1016/j.bbrc.2008.12.163

Zhu, M., Chen, G., Zhang, J., Zhang, Y., Xie, Q., Zhao, Z., et al. (2014). The abiotic stress-responsive NAC-type transcription factor SINAC4 regulates salt and drought tolerance and stress-related genes in tomato (Solanum lycopersicum). Plant Cell Rep. 33, 1851-1863. doi: 10.1007/s00299-014-1662-z

Conflict of Interest Statement: The authors declare that the research was conducted in the absence of any commercial or financial relationships that could be construed as a potential conflict of interest.

Copyright (c) 2015 Ha, Watanabe, Tran, Le, Tanaka, Nguyen, Seki, Nguyen and Tran. This is an open-access article distributed under the terms of the Creative Commons Attribution License (CC BY). The use, distribution or reproduction in other forums is permitted, provided the original author(s) or licensor are credited and that the original publication in this journal is cited, in accordance with accepted academic practice. No use, distribution or reproduction is permitted which does not comply with these terms. 